University of Nebraska - Lincoln

DigitalCommons@University of Nebraska - Lincoln

\title{
$4-2011$
}

\section{High-Resolution Studies of Domain Switching Behavior in Nanostructured Ferroelectric Polymers}

\author{
Pankaj Sharma \\ University of Nebraska-Lincoln, psharma@huskers.unl.edu \\ Timothy J. Reece \\ University of Nebraska-Lincoln, reecetj@unk.edu \\ Stephen Ducharme \\ University of Nebraska-Lincoln, sducharme1@unl.edu \\ Alexei Gruverman \\ University of Nebraska-Lincoln, agruverman2@unl.edu
}

Follow this and additional works at: https://digitalcommons.unl.edu/physicsducharme

Part of the Physics Commons

Sharma, Pankaj; Reece, Timothy J.; Ducharme, Stephen; and Gruverman, Alexei, "High-Resolution Studies of Domain Switching Behavior in Nanostructured Ferroelectric Polymers" (2011). Stephen Ducharme Publications. 76.

https://digitalcommons.unl.edu/physicsducharme/76

This Article is brought to you for free and open access by the Research Papers in Physics and Astronomy at DigitalCommons@University of Nebraska - Lincoln. It has been accepted for inclusion in Stephen Ducharme Publications by an authorized administrator of DigitalCommons@University of Nebraska - Lincoln. 


\title{
High-Resolution Studies of Domain Switching Behavior in Nanostructured Ferroelectric Polymers
}

\author{
Pankaj Sharma, Timothy J. Reece, Stephen Ducharme, and Alexei Gruverman
}

Department of Physics and Astronomy and Nebraska Center for Materials and Nanoscience, University of Nebraska-Lincoln, Lincoln, Nebraska 68588-0299, USA

Corresponding author - A. Gruverman, email agruverman2@unl.edu

\begin{abstract}
We have demonstrated an effective electrical control of polarization in the individual crystalline nanomesas of the ferroelectric polymer, poly(vinylidene fluoride)-trifluoroethylene (PVDF-TrFE) and its relation to the polymer structure. The mechanism of polarization reversal has been investigated via sub-10 $\mathrm{nm}$ real space imaging of domain pattern evolution under an applied electric field. The domain switching behavior revealed in PVDF-TrFE nanomesas is drastically different from that observed in inorganic solid-state crystalline ferroelectrics. The nanoscale features of the switching process include remote domain nucleation and spatially nonuniform wall velocity. Local switching spectroscopy and domain dynamics studies relate the observed switching features to a random-bond type disorder associated with defects in conformation and molecular packing.
\end{abstract}

Keywords: Ferroelectric polymer, disorder, domain switching, piezoresponse force microscopy
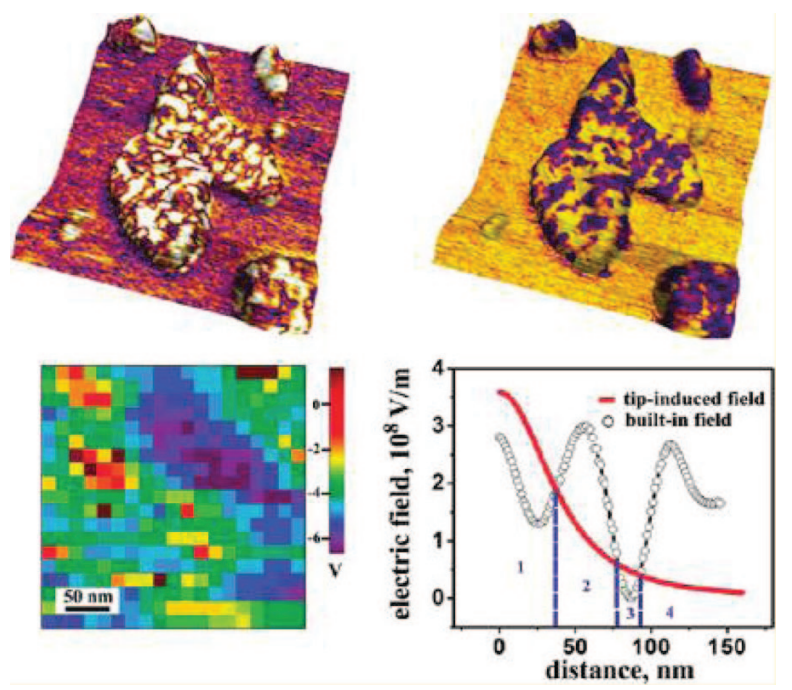

For decades, because of its fundamental and technological importance, the switching behavior of ferroelectrics has been a focal point of experimental and theoretical studies. ${ }^{1}$ Domain nucleation and wall motion are two basic mechanisms involved in electrically induced polarization switching. ${ }^{2}$ Knowledge of the field-time dependency of the nucleation rate and domain wall velocity are critical for predicting the switching performance of ferroelectric-based electronic devices ranging from ferroelectric random access memory chips to field effect transistors. There is a large amount of experimental evidence that both switching mechanisms are strongly affected by the defect structure of the ferroelectric medium. ${ }^{3,4}$ Domain wall creep has been observed in the disordered systems with longrange variations in pinning potential (so-called random field disorder) with the wall velocity obeying an exponential field dependence, while the short-range (random bond) disorder leads to a power law dependence. ${ }^{5,6}$ Dissimilar distribution functions of switching times in ferroelectric films with different microstructure are the root cause for qualitatively different time-dependent switching behavior described either by the statistical Kolmogorov-Avrami-Ishibashi model ${ }^{7,8}$ or nucleation limited kinetics. ${ }^{9}, 10$ In the case of ferroelectric nanostructures, the switching behavior is further complicated by a delicate balance between surface and bulk energy, e.g., interfacial strain, asymmetry of electrical boundary conditions, surface depolarization energy, and so forth. Electron and scanning probe microscopy studies made a crucial contribution toward compre- hensive understanding of the microstructural aspects of the ferroelectric switching. ${ }^{11}$ Particularly, piezoresponse force microscopy (PFM) has been instrumental in providing a direct experimental evidence on the interplay between microstructural features and nucleation and domain growth kinetics, allowing for well-justified interpretation of experimental results. In this paper, we carry out PFM investigation of local polarization reversal in ferroelectric copolymer, poly(vinylidene fluoride)trifluoroethylene (PVDF-TrFE), nanostructures with sub-10 $\mathrm{nm}$ lateral resolution. The nanoscale switching mechanism revealed in this material system is drastically different from that observed in inorganic crystalline ferroelectrics. It is shown that the observed features are related to the extreme spatial nonuniformity of a local field caused by the structural variations in polymer medium.

Among synthetic polymers, PVDF-TrFE exhibits the highest piezoelectric activity, which determines its wide application in piezoelectric transducers and sensors. ${ }^{12,13}$ A relatively high value of the spontaneous polarization, and a possibility of fabricating crystalline nanostructures have led to consideration of PVDF-TrFE for all-organic memory and mass data storage devices. ${ }^{14-17}$ Discovery of ferroelectricity in ultrathin films of PVDF-TrFE ${ }^{18}$ has triggered the fundamental studies of critical scaling behavior in ferroelectric structures. On the other hand, the varying degree of crystallinity makes the PVDF-TrFE a model material for investigation of the role of structural fluctuations in polarization switching. 
On the molecular level, ferroelectric polarization in PVDFTrFE arises from alignment of molecular dipoles formed by positive hydrogen ions and negative fluorine ions. ${ }^{19}$ The alltrans ( $\beta$-phase) molecular conformation results in the dipoles being oriented perpendicular to the chain axis and polarization reversal is associated with rotation of these dipoles about the molecular chain. In the crystalline $\beta$-phase, molecules tend to pack parallel to each other forming a quasi-hexagonal orthorhombic structure with lattice spacing of approximately $0.5 \mathrm{~nm}$ along the polar axis. Polarization reversal studies by means of macroscopic dielectric measurements and, most recently, by local probe techniques revealed a rather complex switching behavior that could not be fully explained neither by KAI nor by nucleation limited models implying a strong dependence of the switching potential on defect structure. ${ }^{20-24}$

Over the span of just several years PFM has become a primary method to probe the static and dynamic properties of the ferroelectric materials at the nanoscale. ${ }^{25}$ However, very few reports have succeeded in unambiguous high-resolution detection of polarization dynamics in PVDF-TrFE films as the PFM signal is strongly influenced by the presence of amorphous phase, complex morphology and can additionally be affected by morphological instability as a result of electrically induced decomposition of a polymer sample. ${ }^{26}$ Improved crystallinity of the PVDF-TrFE samples used in this study and application of the resonance-enhanced PFM mode allowed us to delineate the polarization distribution and investigate the local switching behavior with the sub-10 $\mathrm{nm}$ spatial resolution that is rarely attainable in ferroelectric polymers.

In this study, highly crystalline ultrathin samples of PVDFTrFE with a molar content ratio of 80:20 were fabricated on highly doped (0.001-0.005 $\left.\Omega^{*} \mathrm{~cm}\right)$ silicon substrates using the horizontal Schaefer variation of the Langmuir-Blodgett (LB) transfer technique. Langmuir layers were dispersed on an ultrapure water subphase from a $0.05 \%$ solution of PVDF-TrFE in dimethyl sulfoxide and then transferred to the Si substrate by horizontal dipping. Deposition was carried out at a constant surface pressure of $5 \mathrm{mN} / \mathrm{m}$ and temperature of $25^{\circ} \mathrm{C}$. Film thickness was controlled by the number of deposition steps. After deposition, the films were annealed at $135^{\circ} \mathrm{C}$ for a period of $90 \mathrm{~min}$ with subsequent cooling to room temperature at a rate of $1{ }^{\circ} \mathrm{C} / \mathrm{min}$ in a microprocessor-controlled forced air oven. Further details of film preparation can be found elsewhere. ${ }^{27}$

A commercial atomic force microscope (Asylum MFP-3D) was used in this study to implement single-frequency PFM as well as a dual AC resonance tracking PFM (DART-PFM) mode $^{28}$ to get $2 \mathrm{D}$ maps of the local switching parameters. Domain visualization has been performed by applying a highfrequency modulating voltage $(400-800 \mathrm{kHz}, 1.0-1.5 \mathrm{~V})$ using Pt-Ti-coated silicon (Mikromasch) and Au-coated SiN tips (Olympus). Resonance frequency varied by about $10 \mathrm{kHz}$ due to variations in surface morphology. The presence of as-grown domains did not have any effect on the resonance, while a significant change $(\sim 20 \mathrm{kHz})$ was observed for newly switched domains.

Atomic force microscopy (AFM) studies showed that the three monolayer (ML) PVDF-TrFE samples exhibited not a continuous morphology but an island-type structure consisting of irregularly shaped nanomesas formed via self-assembling. ${ }^{29}$ Figure 1a shows a topographic image of a typical nanomesa of approximately $200-300 \mathrm{~nm}$ in lateral size and 25-30 $\mathrm{nm}$ in thickness. Corresponding PFM amplitude and phase
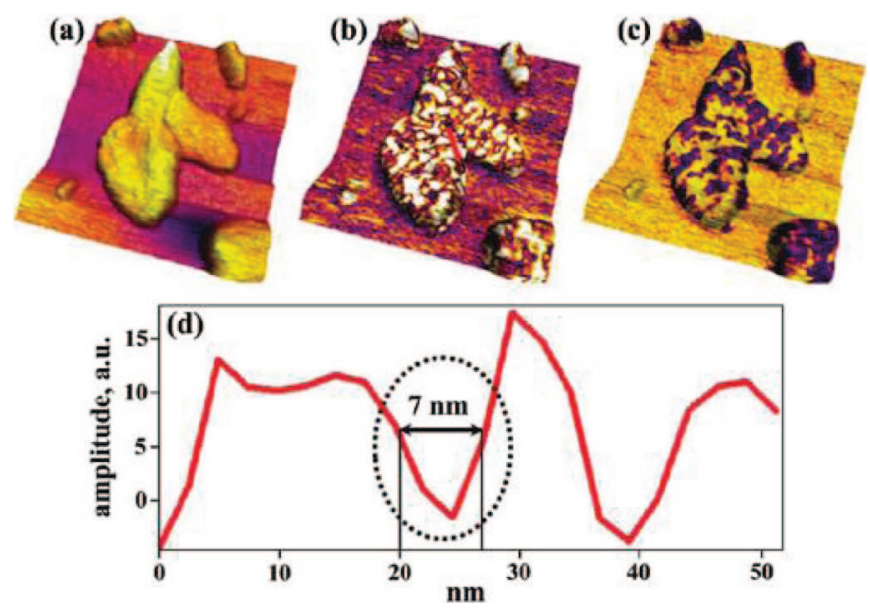

Figure 1. (a) Topographic image of an individual PVDF-TrFE nanomesa; (b,c) corresponding PFM amplitude (b) and phase (c) images superimposed on the topographic image. Image size is $600 \times$ $600 \mathrm{~nm}^{2}$. (d) Cross-section along the red line in the PFM amplitude image. Domain wall width is determined as a trough width in the cross-section profile between two adjacent maxima corresponding to two antiparallel domains (full width at the half-maximum).

images overlaid on the topographic image are shown in Figure $1 \mathrm{~b}, \mathrm{c}$, respectively. It can be seen that the as-grown nanomesa is in a polydomain state with an average domain size in the range of $30-40 \mathrm{~nm}$, which is consistent with the previously reported values. ${ }^{30} \mathrm{~A}$ strong PFM amplitude signal suggests that the nanomesas are composed of molecular chains aligned parallel to the substrate so that there is a strong out-of-plane polarization component. This is in agreement with the predominant [110] crystalline orientation determined from the XRD studies of nanomesas arrays. ${ }^{29}$ In the PFM amplitude image (Figure $1 b$ ), domain walls appear as narrow dark lines with a characteristically weak signal. Location of the domain walls does not coincide with location of the morphological features in the topography image. This suggests that features in the topography image do not necessarily indicate the presence of the grain boundaries or discontinuous PVDF-TrFE chains. Cross-section analysis of the PFM amplitude map (Figure 1d) shows that the width of the domain wall image is about $7 \mathrm{~nm}$ illustrating the sub- $10 \mathrm{~nm}$ lateral resolution achieved in our studies.

In PFM, control of polarization can be realized on a relatively large (global) or on a very local (nanometer) scale. ${ }^{31} \mathrm{In}$ the former case, the electrically biased tip is scanned over a selected area in the range from several to tens of square micrometers. ${ }^{26,32}$ Provided that a tip-generated field exceeds the local threshold field, polarization reversal in the scanned area can be achieved. The efficacy of this type of polarization control in the individual PVDF-TrFE nanomesas is illustrated in Figures $2 a-c$ and $3 a-c$. Scanning over the nanomesas with the tip held under a dc bias of several volts transforms their initially polydomain structure into a single domain state.

Local polarization control is typically accomplished by positioning the tip at a selected location and applying voltage pulses of varying duration and amplitude. Localized field application leads to nucleation of a single domain, which further expands via lateral domain wall motion. This approach has been extensively used over the last several years for investigation of the nanoscale mechanism of polarization reversal by measuring the time-field dependence of the growing domain size. ${ }^{33-35}$ 

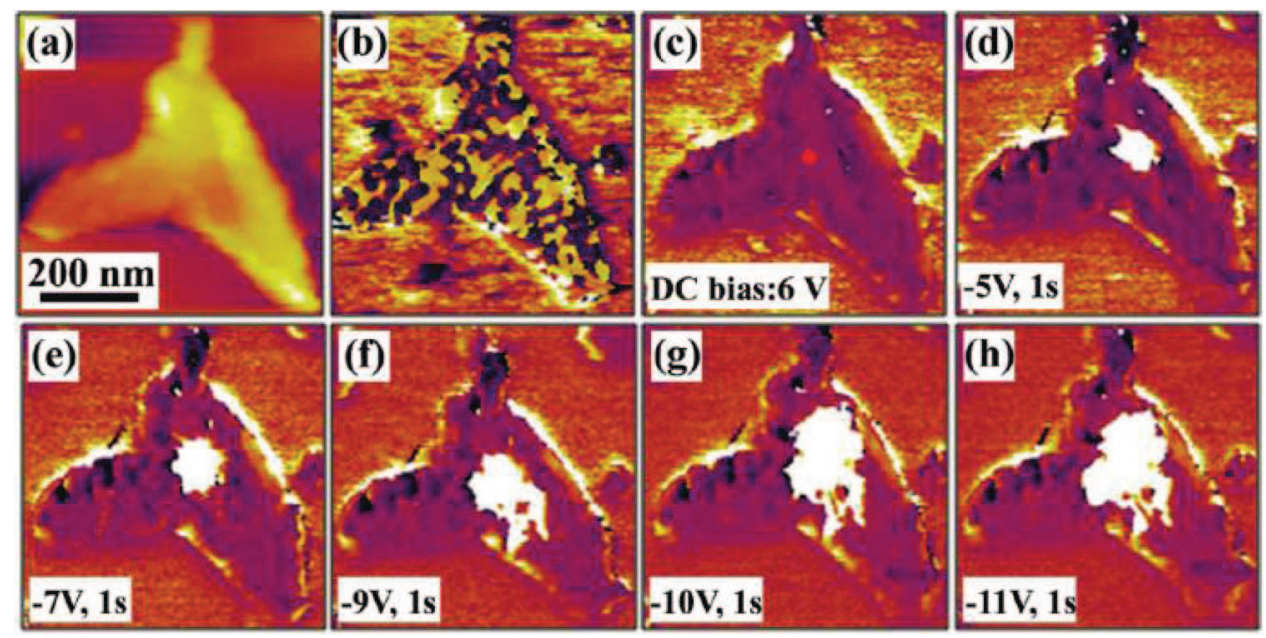

Figure 2. (a) Topographic image of the PVDF-TrFE nanomesa and (b) corresponding PFM phase image of the as-grown domain structure; (c) PFM phase image of the same nanomesa after global switching under a dc bias of $6 \mathrm{~V}$; $(\mathrm{d}-\mathrm{h})$ voltage-dependent growth of a nucleated domain as a result of a local voltage pulse application (tip location is marked by the red dot).

Figure $2 \mathrm{~d}-\mathrm{h}$ illustrates a process of local polarization reversal induced by voltage pulses with an incrementally increasing amplitude and a fixed duration. Formation of a stable domain (with a minimum size of about $20-30 \mathrm{~nm}$ ) and its lateral growth has been detected when the voltage exceeds $4 \mathrm{~V}$, which corresponds to the threshold field of approximately $2 \times 10^{8} \mathrm{~V} / \mathrm{m}$, in agreement with the previously reported values. ${ }^{36}$ In contrast to the local PFM switching observed in crystalline inorganic ferroelectrics, ${ }^{6,} 33,37$ the growing domain in the PVDF-TrFE nanomesa acquires a profoundly irregular shape, which can be an indication of a high degree of nonuniformity in the local switching potential. Because of this feature, extraction of a precise value for the wall velocity from the obtained data is difficult. For this reason, as an alternative approach to modeling the voltage-dependent switching dynamics, we considered the field dependence of the switched domain area $S$ (Figure 4a).

In our calculations, the probe is modeled as a charged sphere of radius $R$, at a distance $\delta$ above the sample surface. ${ }^{38}$ The distribution of the probe-generated normal component of the electric field $E$ as a function of distance $r$ from the tip on sample surface is given as

$$
E(r)=\frac{1}{2 \pi \varepsilon_{0}}\left(\frac{C_{\mathrm{t}} V_{\mathrm{t}}}{\sqrt{\varepsilon_{\mathrm{a}} \varepsilon_{\mathrm{c}}}+1}\right) \sqrt{\frac{\varepsilon_{\mathrm{a}}}{\varepsilon_{\mathrm{c}}}\left[(R+\delta)^{2}+r^{2}\right]^{3 / 2}}
$$

where $C_{t}$ and $V_{t}$ are the tip capacitance and bias respectively, and $\varepsilon_{\mathrm{a}}$ and $\varepsilon_{\mathrm{c}}$ are the dielectric constants of PVDF-TrFE along the polar and the nonpolar axis. We have used the following values for field calculation: $V_{\mathrm{t}}=5 \mathrm{~V}, R=50 \mathrm{~nm}, \delta=1 \mathrm{~nm}, \varepsilon_{\mathrm{a}}=$ 4 , and $\varepsilon_{\mathrm{c}}=10$. Tip capacitance has been estimated to be $C_{\mathrm{t}}=12$ $\times 10^{-17} \mathrm{~F}$.

The field dependence of the switched area has been fitted by a stretched exponential function $S \sim \exp \left(-\left(E_{0} / E\right)^{\mu}\right)$ (where $E_{0}$ is the threshold field and $\mu$ is a dynamical exponent). Approximating the switched domain area by equiareal circles, for a fixed pulse duration the wall velocity averaged over all azimuthal directions can be estimated as $v \sim S^{1 / 2}$. This analysis yielded $\mu \approx 0.26$ indicating the random bond regime of domain switching.

A mechanism of polarization reversal in PVDF-TrFE involves rotation of $\mathrm{CH}_{2}-\mathrm{CF}_{2}$ and $\mathrm{CHF}-\mathrm{CF}_{2}$ dipoles about the $\mathrm{C}-\mathrm{C}$ main chain with domain wall progressing via molecular chain rotations in the $a-b$ plane of the crystalline lamella. ${ }^{39}$
This mechanism entails a strong effect of the chain azimuthal orientation on the anisotropy of the domain wall velocity. Clearly, the switching process should be also strongly affected by the degree of sample crystallinity. As the sample has been annealed at $135{ }^{\circ} \mathrm{C}$ and slowly cooled down to room temperature it is natural to expect an almost $100 \%$ crystallization. However, even if this would be the case, polarization relaxation observed in the ferroelectric $\beta$-phase ${ }^{40}$ suggests a high degree of disorder associated with defects in conformation and molecular packing developing during phase transition.

The disorder in PVDF-TrFE produces an even more unconventional effect on the time-dependent behavior of the switching process illustrated in Figure $3(d-h)$. Here, the domain growth is visualized as a function of the pulse duration for the fixed pulse amplitude of $-5 \mathrm{~V}$. It can be seen that the switching proceeds not only through the lateral expansion of a nucleated domain underneath the tip (parent domain), but also through nucleation of a number of domains away from the tip-surface contact point, an effect which will be referred to as "remote nucleation". The time dependence of the switched domain area depicts an essentially nonsteady process with considerable variations (jumps) in the rate of switching (Figure $4 b, c)$. Comparison of these plots with the PFM switching images shows that these jumps are associated with remote nucleation events occurring upon an increase in the pulse duration after the parent domain growth comes to a halt. The remote nucleation effect is likely a result of strong variations in a local potential caused by the structural variations in the polymer medium. Note that in the typical experimental conditions, the electric field due to the biased PFM probe extends over the distance of up to several hundred nanometers from the tip-sample contact. Thus, although the walls of the growing parent domain can be pinned at the local energy minimum, nucleation of new domains is still possible at more distant locations provided that the pulse duration is longer than the nucleation time for the local value of the tip-generated field. In the framework of the Landauer model, ${ }^{41}$ the nucleation time can be found from $t=t_{\infty} \exp \left[\left(U_{\mathrm{a}} / k_{\mathrm{B}} T\right)\left(E_{\mathrm{a}} / E\right)\right]$, where $k_{\mathrm{B}}$ is the Boltzmann constant, $T$ is temperature, and $U_{\mathrm{a}}$ is the energy barrier for domain nucleation. The value of $t_{\infty}$ extracted from the global switching data (e.g., Reference 21) is typically in the 2-5 ns range. Thus, the energy barrier for nucleation can be es- 


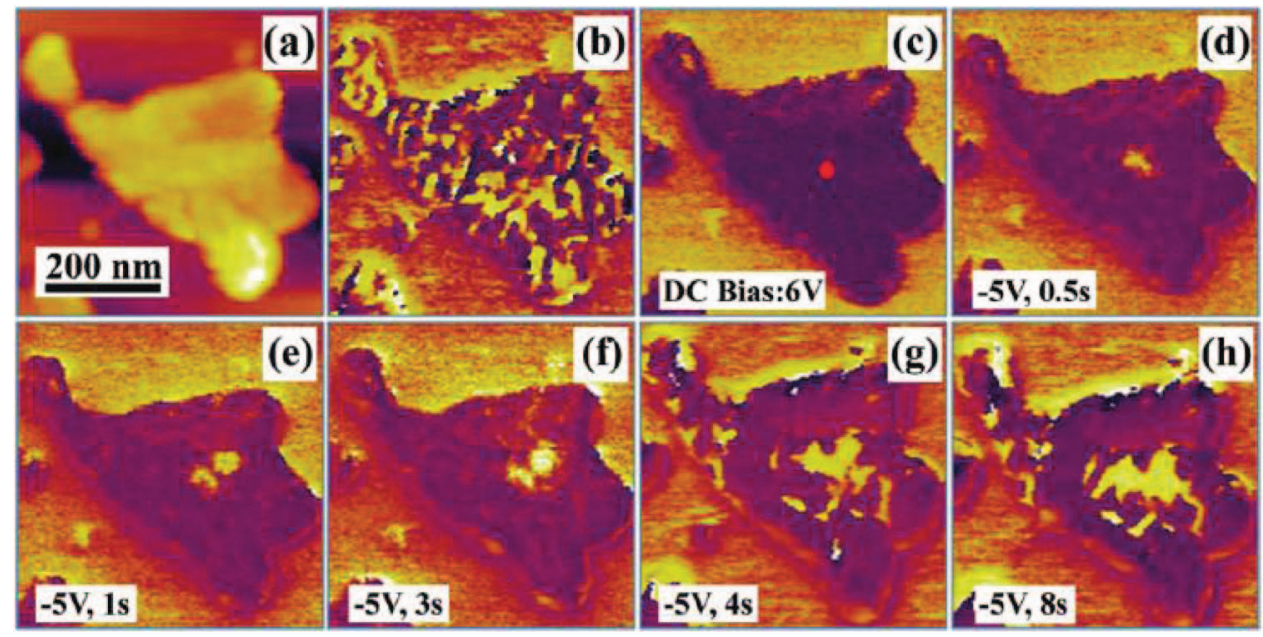

Figure 3. (a) Topographic image of the PVDF-TrFE nanomesa and (b) corresponding PFM phase image of the as-grown domain structure; (c) PFM phase image of the same nanomesa after global switching under a dc bias of $6 \mathrm{~V}$; $(\mathrm{d}-\mathrm{h})$ time-dependent switching of polarization as a result of a local voltage pulse application (tip location is marked by the red dot).
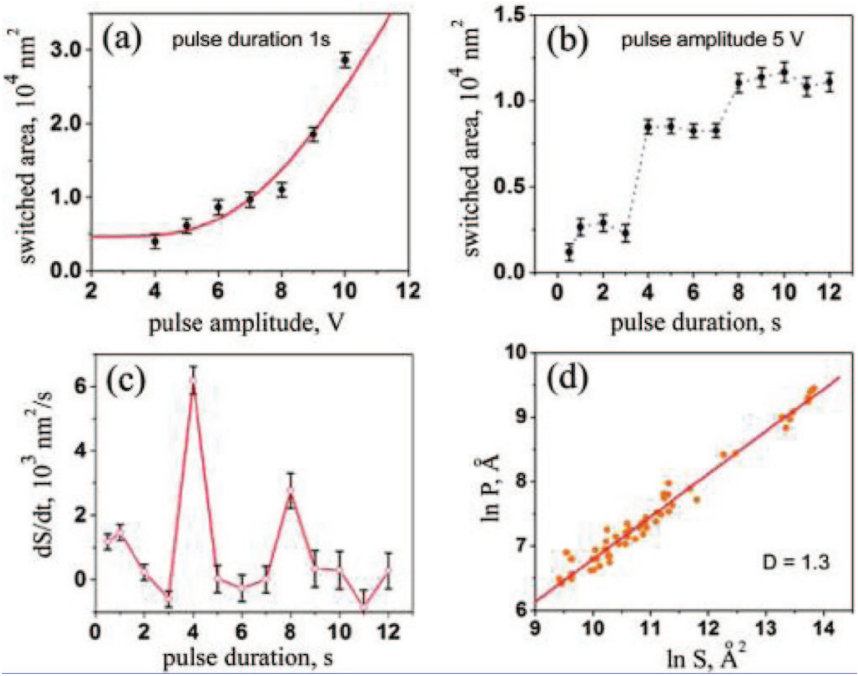

Figure 4. (a) Bias dependence of the total domain switchKed area. (b) Time dependence of the total domain switched area. (c) $\mathrm{d} S / \mathrm{d} t$ as a function of the pulse duration illustrating a highly nonsteady switching rate. (d) Perimeter of the switched domains as a function of the total domain area. The slope of the log-log plot (orange line) is related to the fractal dimension, $P=K^{*} S^{D / 2}$.

timated as $0.4 \mathrm{eV}$. Given that nucleation times in our studies are in the range from 0.5 to $15 \mathrm{~s}$, local nucleation potential varies by about $9 \times 10^{-2} \mathrm{eV}$, or over $20 \%$, a signature of a highly nonuniform medium.

Geometrical aspects of domain growth have been studied by performing fractal analysis of the time-evolving domain patterns (Figure $4 \mathrm{~d}$ ). ${ }^{42}$ Fractal analysis provides a simple description of the effect of spatial correlations in a disordered medium on polarization reversal. In this context, lateral domain growth is viewed as a flow of dipole rotations in the randomly distributed internal potential, which forms a random percolating network. The fractal dimension (or Hausdorff dimension) of the underlying fractal structure then represents the spatial disorder. The fractal dimension $D$ has been determined from the $\log -\log$ plot of the domain perimeter $P$ versus the area $S$ of the switched domains (Figure $4 \mathrm{~d}$ ). ${ }^{43}$ Fitting this
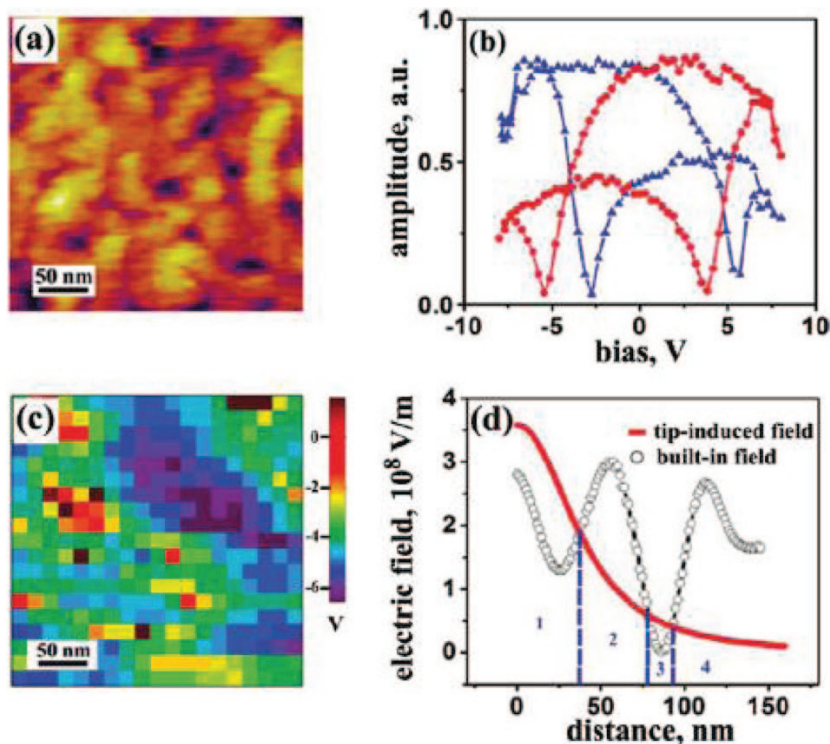

Figure 5. (a) Surface topography, (b) representative local hysteresis loops, (c) SS-PFM map of the negative threshold bias, and (d) tip-induced field as function of the distance from the contact point and a cross-section profile of the negative threshold field.

dependence by $P=K^{*} S^{D / 2}$ yields a value $D=1.32$. The fractal dimension can be viewed as the degree of roughness of a boundary between two phases. For an intersection of such a boundary with the sample surface, $D$ should vary between 1 and 2 (with 1 corresponding to a smooth curve with a continuous derivative and 2 to a very rough surface-filling curve) ${ }^{44}$ The obtained $D$ value is lower than that reported for $\mathrm{BiFeO}_{3}$ and $\mathrm{Pb}(\mathrm{Zr}, \mathrm{Ti}) \mathrm{O}_{3}$ films, which can be explained if one assumes that the correlation length in polymer PVDF-TrFE nanomesas is shorter in comparison to solid-state crystalline ferroelectrics. $^{45,46}$

Further insight into the disorder potential has been obtained by mapping the spatial distribution of the local switching parameters using the DART-PFM mode (Figure 5). ${ }^{47}$ This method involves point-by-point acquisition of the local hysteresis loops over a grid of $20 \times 20$ points on the surface area of 
$300 \times 300 \mathrm{~nm}^{2}$ (Figure 5a,b). Subsequent numerical analysis of the obtained loops allows extraction of the local switching parameters, such as threshold bias, imprint and remanent polarization, which can be represented in the form of 2D maps.

The variable color map of the local threshold bias in Figure $5 c$ can be considered as a manifestation of strong nonuniformity of the internal potential. Correlation analysis of the switching threshold map shows that the internal potential $U_{\text {th }}$ profile varies by several Volts over the characteristic length of $\sim 50 \mathrm{~nm}$. This estimate allows comparison of the local potential distribution with the observed spatial variations in the switching kinetics (Figure 3). In Figure $5 d$, the spatial profile of the normal component of the tip-generated field calculated according to Equation 1 is overlaid on the cross-section of the negative threshold field from Figure 5 c calculated as $U_{\text {th }} / d$, where $d$ is PVDF-TrFE thickness. It can be seen that within the radius of $35 \mathrm{~nm}$ from the tip-sample contact (region 1), the electric field generated by the tip is high enough to induce the polarization reversal. However, in the region within a distance range from 35 to $75 \mathrm{~nm}$ (region 2), where the threshold field for switching is higher than the applied field, the switching should be suppressed. In region 3, which ranges from 75 to 90 $\mathrm{nm}$ from the tip, the applied electric field is again high enough to induce polarization reversal which would result in domain nucleation at some distance from the parent domain as is observed in Figure 3. At even larger distances exceeding $90 \mathrm{~nm}$ (region 4), no switching can occur as the tip-induced field is too low. Therefore, spatially resolved spectroscopic analysis in Figure $5 d$ is consistent with the interpretation of the remote nucleation effect as a result of strong variations in the local potential in the disordered polymer medium.

In summary, we have demonstrated the sub-10 nm spatial resolution in depicting the switching behavior in the individual crystalline PVDF-TrFE nanomesas. Local polarization reversal proceeds via formation of profoundly irregular domains and remote domain nucleation suggesting strong variations in the local potential. Nanoscale local spectroscopy and domain switching studies allow consistent interpretation of the observed switching behavior, which is related to a random-bond type disorder associated with defects in conformation and molecular packing. Fractal analysis of the evolving domain patterns yields the fractal dimension lower than that reported for solid-state crystalline ferroelectrics, which can be explained by a shorter correlation length in polymer PVDFTrFE nanomesas.

Acknowledgment - This research was supported by the U.S. Department of Energy, Office of Basic Energy Sciences, Division of Materials Sciences and Engineering under Award DE-SC0004530 and by Nebraska Research Initiative.

Supporting Information - One additional figure follows the References.

\section{References}

1. Scott, J. F. Ferroelectric memories; Springer: Berlin, 2000.

2. Fatuzzo, E.; Merz, W. J. Ferroelectricity; North-Holland Publishing Company: Amsterdam, 1967.

3. Scott, J. F.; McMillan, L. D.; Araujo, C. A.Switching kinetics of lead zirconate titanate sub-micron thin-film memories J. Appl. Phys. 1988, 64, 787
4. Jesse, S.; Rodriguez, B. J.; Choudhury, S.; Baddorf, A. P.; Vrejoiu, I.; Hesse, D.; Alexe, M.; Eliseev, E. A.; Morozovska, A. N.; Zhang, J.; Chen, L. Q.; Kalinin, S. V. Direct imaging of the spatial and energy distribution of nucleation centres in ferroelectric materials Nat. Mater. 2008, 7, 209

5. Jo, J. Y.; Yang, S. M.; Kim, T. H.; Lee, H. N.; Yoon, J.-G.; Park, S.; Jo, Y.; Jung, M. H.; Noh, T. W. Nonlinear dynamics of domainwall propagation in epitaxial ferroelectric thin films Phys. Rev. Lett. 2009, 102, 045701

6. Tybell, T.; Paruch, P.; Giamarchi, T.; Triscone, J. M. Domain wall creep in epitaxial ferroelectric $\mathrm{Pb}\left(\mathrm{Zr}_{0.2} \mathrm{Ti}_{0.8}\right) \mathrm{O}_{3}$ thin films Phys. Rev. Lett. 2002, 89, 097601

7. Ishibashi., Y.; Takagi, Y. Ferroelectric domain switching J. Phys. Soc. Jpn. 1971, 31, 506

8. Kolmogorov, A. N. On the statistical theory of the crystallization of metals Izv. Akad. Nauk USSR, Ser. Math. 1937, 3, 355

9. Du, X. F.; Chen, I. W. Model experiments on fatigue of $\mathrm{Pb}\left(\mathrm{Zr}_{0.53} \mathrm{Ti}_{0.47}\right) \mathrm{O}_{3}$ ferroelectric thin films Appl. Phys. Lett. 1998, 72,1923

10. Tagantsev, A. K.; Stolichnov, I.; Setter, N.; Cross, J. S.; Tsukada, M. Non-Kolmogorov-Avrami switching kinetics in ferroelectric thin films Phys. Rev. B 2002, 66, 214109

11. Rodriguez, B. J.; Jesse, S.; Baddorf, A. P.; Zhao, T.; Chu, Y.-H.; Ramesh, R.; Eliseev, E. A.; Morozovska, A. N.; Kalinin, S. V. Spatially resolved mapping of ferroelectric switching behavior in self-assembled multiferroic nanostructures: strain, size, and interface effects Nanotechnology 2007, 18, 405701

12. Lovinger, A. J. Ferroelectric polymers Science 1983, 220, 1115

13. Lang, S. B.; Muensit, S. Review of some lesser-known applications of piezoelectric and pyroelectric polymers Appl. Phys. A $2006,85,125$

14. Hu, Z.; Tian, M.; Nysten, B.; Jonas, A. M. Regular arrays of highly ordered ferroelectric polymer nanostructures for nonvolatile low-voltage memories Nat. Mater. 2009, 8, 62

15. Park, Y. J.; Kang, S. J.; Lotz, B.; Brinkmann, M.; Thierry, A.; Kim, K. J.; Park, C. Ordered ferroelectric PVDF-TrFE thin films by high throughput epitaxy for nonvolatile polymer memory Macromolecules 2008, 41, 8648

16. Ducharme, S.; Gruverman, A. Ferroelectrics start the presses Nat. Mater. 2009, 8, 9

17. El-Hami, K.; Hara, M.; Yamada, H.; Matsushige, K. The use of nanotechnology to fabricate ultra-high density molecular memory in $\mathrm{P}(\mathrm{VDF} / \mathrm{TrFE})$ copolymer: data storage Ann. Chim. Sci. Mat. 2001, 26, 217

18. Ducharme, S.; Fridkin, V. M.; Bune, A. V.; Palto, S. P.; Blinov, L. M.; Petukhova, N. N.; Yudin, S. G. Intrinsic ferroelectric coercive field Phys. Rev. Lett. 2000, 84, 175

19. Furukawa, T. Ferroelectric properties of vinylidene fluoride copolymers Phase Transitions 1989, 18, 143

20. Kliem, H.; Tadros-Morgane, R. Extrinsic versus intrinsic ferroelectric switching: experimental investigations using ultrathin PVDF Langmuir-Blodgett films J. Phys. D: Appl. Phys. 2005, 38, 1860

21. Gysel, R.; Stolichnov, I.; Tagantsev, A. K.; Setter, N.; Mokry, $P$. Restricted domain growth and polarization reversal kinetics in ferroelectric polymer thin films J. Appl. Phys. 2008, 103, 084120

22. Bystrov, V. S.; Bdikin, I. K.; Kiselev, D. A.; Yudin, S.; Fridkin, V. M.; Kholkin, A. L. Nanoscale polarization patterning of ferroelectric Langmuir-Blodgett P(VDF-TrFE) films J. Phys. D: Appl. Phys. 2007, 40, 4571

23. Gaynutdinov, R. V.; Lysova, O. A.; Yudin, S. G.; Tolstikhina, A. L.; Kholkin, A. L.; Fridkin, V. M.; Ducharme, S. Polarization switching kinetics of ferroelectric nanomesas of vinylidene fluoride-trifluoroethylene copolymer Appl. Phys. Lett. 2009, 95, 023303 
24. Kim, Y.; Kim, W.; Choi, H.; Hong, S.; Ko, H.; Lee, H.; No, K. Nanoscale domain growth dynamics of poly(vinylidene fluoride-co-trifluoroethylene) thin films App. Phys. Lett. 2010, 96, 012908

25. Bonnell, D. A.; Kalinin, S. V.; Kholkin, A. L.; Gruverman, A. Piezoresponse force microscopy: A window into electromechanical behavior at the nanoscale MRS Bull. 2009, 34, 648

26. Rodriguez, B. J.; Jesse, S.; Kalinin, S. V.; Kim, J.; Ducharme, S.; Fridkin, V. M. Nanoscale polarization manipulation and imaging of ferroelectric Langmuir-Blodgett polymer films Appl. Phys. Lett. 2007, 90, 122904

27. Sorokin, A.; Palto, S.; Blinov, L.; Fridkin, V. M.; Yudin, S. Ultrathin ferroelectric Langmuir-Blodgett films Mol. Mater. 1996, 6,61

28. Rodriguez, B. J.; Callahan, C.; Kalinin, S. V.; Proksch, R. Dualfrequency resonance-tracking atomic force microscopy Nanotechnology 2007, 18, 475504

29. Bai, M.; Ducharme, S. Ferroelectric Nanomesa Formation from Polymer Langmuir-Blodgett Films Appl. Phys. Lett. 2004, 85, 3528

30. Sharma, P.; Reece, T.; Wu, D.; Fridkin, V. M.; Ducharme, S.; Gruverman, A. Nanoscale domain patterns in ultrathin polymer ferroelectric films J. Phys.: Condens. Matter 2009, 21, 485902

31. Gruverman, A.; Kalinin, S. V. Piezoresponse force microscopy and recent advances in nanoscale studies of ferroelectrics $J$. Mat. Sci. 2006, 41, 107

32. Nath, R.; Chu, Y.-H.; Polomoff, N. A.; Ramesh, R.; Huey, B. D. High speed piezoresponse force microscopy: $<1$ frame per second nanoscale imaging App. Phys. Lett. 2008, 93, 072905

33. Rodriguez, B. J.; Nemanich, R. J.; Kingon, A.; Gruverman, A.; Kalinin, S. V.; Terabe, K.; Liu, X. Y.; Kitamura, K. Domain growth kinetics in lithium niobate single crystals studied by piezoresponse force microscopy Appl. Phys. Lett. 2004, 86, 012906

34. Pertsev, N. A.; Petraru, A.; Kohlstedt, H.; Waser, R.; Bdikin, I. K.; Kiselev, D.; Kholkin, A. L. Dynamics of ferroelectric nanodomains in $\mathrm{BaTiO}_{3}$ epitaxial thin films via piezoresponse force microscopy Nanotechnology 2008, 19, 375703

35. Kalinin, S. V.; Morozovska, A. N.; Chen, L. Q.; Rodriguez, B. J. Local polarization dynamics in ferroelectric materials Rep. Prog. Phys. 2010, 73, 056502
36. Bystrov, V. S.; Bystrova, N. K.; Paramonova, E. V.; Vizdrik, G.; Sapronova, A. V.; Kuehn, M.; Kliem, H.; Kholkin, A. L. First principle calculations of molecular polarization switching in P(VDF-TrFE) ferroelectric thin Langmuir-Blodgett films $J$. Phys.: Condens. Matter 2007, 19, 456210

37. Rosenman, G.; Urenski, P.; Agronin, A.; Rosenwaks, Y.; Molotskii, M. Submicron ferroelectric domain structures tailored by high-voltage scanning probe microscopy Appl. Phys. Lett. 2003, 82, 103

38. Mele, E. J. Screening of a point charge by an anisotropic medium: Anamorphoses in the method of images Am. J. Phys. 2001, 69, 557

39. Furukawa, T.; Nakajima, T.; Takahashi, Y. Factors governing ferroelectric switching characteristics of thin VDF/TrFE copolymer films IEEE Trans. Dielectr. Electr. Insul. 2006, 13, 1120

40. Rodriguez, B. J.; Jesse, S.; Kim, J.; Ducharme, S.; Kalinin, S. V. Local probing of relaxation time distributions in ferroelectric polymer nanomesas: Time-resolved piezoresponse force spectroscopy and spectroscopic imaging Appl. Phys. Lett. 2008, 92, 232903

41. Landauer, R. Electrostatic considerations in $\mathrm{BaTiO}_{3}$ domain formation during polarization reversal J. Appl. Phys. 1957, 28, 227

42. Nakayama, T.; Yakubo, K. Fractal concepts in condensed matter physics; Springer: Berlin, 2003.

43. Horcas, I.; Fernandez, R.; Gomez-Rodriguez, J. M.; Colchero, J.; Gomez-Herrero, J.; Baro, A. M. WSXM: A software for scanning probe microscopy and a tool for nanotechnology Rev. Sci. Instrum. 2007, 78, 013705

44. Ott, E. Chaos in dynamical systems; Cambridge University Press: New York, 1993.

45. Catalan, G.; Bea, H.; Fusil, S.; Bibes, M.; Paruch, P.; Barthelemy, A.; Scott, J. F. Fractal dimension and size scaling of domains in thin films of multiferroic $\mathrm{BiFeO}_{3}$ Phys. Rev. Lett. 2008, 100, 027602

46. Paruch, P.; Giamarchi, T.; Triscone, J. M. Domain wall roughness in epitaxial ferroelectric $\mathrm{Pb}\left(\mathrm{Zr}_{0.2} \mathrm{Ti}_{0.8}\right) \mathrm{O}_{3}$ thin films Phys. Rev. Lett. 2005, 94, 197601

47. Jesse, S.; Baddorf, A. P.; Kalinin, S. V. Switching spectroscopy piezoresponse force microscopy of ferroelectric materials Appl. Phys. Lett. 2006, 88, 062908 

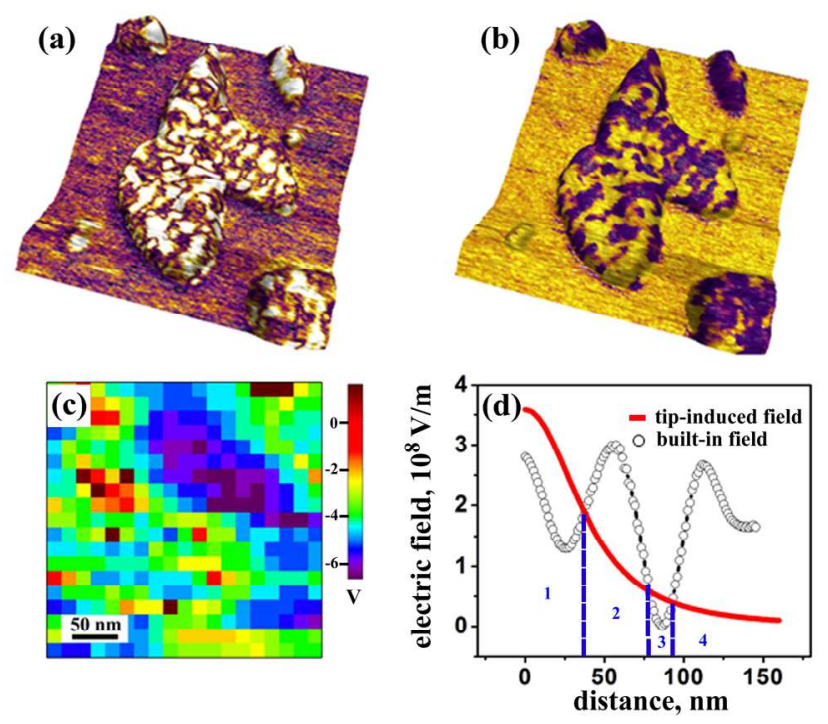

PFM amplitude (a) and phase (b) images of an individual PVDF-TrFE nanomesa superimposed on the topographic image. Image size is $600 \times 600 \mathrm{~nm}^{2}$. (c) A map of the negative threshold bias, (d) tip-induced field as function of the distance from the contact point and cross-section profile of the negative threshold field in. 